\title{
Focused Ion Beam Sample Preparation of Complex Devices
}

\author{
P.E. Russell ${ }^{1,2}$, K.L. Bunker ${ }^{1}$, R. Garcia ${ }^{1}$, T. J. Stark ${ }^{2}$, J.P. Vitarelli ${ }^{2}$ \\ ${ }^{1}$ Analytical Instrumentation Facility and Materials Science and Engineering Dept., North \\ Carolina State University, Box 7531, Raleigh, NC, 27695 \\ ${ }^{2}$ Materials Analytical Services, Inc., 616 Hutton St., Suite 101, Raleigh, NC 27606
}

Focused Ion Beam (FIB) systems can be used for a wide range of demanding sample preparation tasks. The site-specific milling and excellent imaging capabilities of the FIB in combination with the additional features of material deposition, mechanical micromanipulation, gas injection, and selective and chemically enhanced etching of selected materials makes the FIB system an extremely useful and versatile instrument for sample preparation techniques.

A common problem encountered in failure analysis of complex devices is the need for both cross sectional and plan view samples of the same region (i.e. a region containing a unique defect). Recently developed techniques permit lift out of a plan view sample containing one or more site specific features of interest. Utilizing plan view Scanning Transmission Electron Microscopy (STEM), the sample can be fully imaged and characterized to more accurately place the cross sectional prep location, ensuring that the defect is fully contained within the final cross sectional sample. The sample is then returned to the FIB for subsequent lift out of one or more cross sections of the well characterized features of interest. As shown in Figure 1, a plan view sample was lifted from the gate/metal contact of a shorted Field Effect Transistor (FET). A cross-section was then removed from the plan view sample to complete the analysis and demonstrate the successful application of this recently developed lift-out technique.

The backside via problem shown in Figure 2 is another example demonstrating the versatility of FIB in problem solving. After careful removal of the covering layer, an opening was created in a can opener fashion and pried open with the normal lift-out needle of our FIB setup. A residue can clearly be seen in the opening created with the FIB. It is important to note that the use of STEM imaging greatly enhances the ability to perform the initial microscopy on relatively thick samples, which may contain information not obtainable from traditional TEM thin sections.

As a final example, a novel sample preparation technique and custom sample holder for the Hitachi HD-2000 STEM have been designed to allow for a complete and simultaneous STEM-based characterization of fully packaged optoelectronic devices [1]. The STEM-analysis includes the Electron Beam Induced Current (EBIC) and Cathodoluminescence (CL) techniques (Fig. 3 and 4). The sample preparation for this detailed STEM-based analysis requires that electrical contacts must be provided to the sample and the specific region of interest has to be thinned to electron transparency while maintaining full device performance. The preparation process is divided into several steps, including mechanical thinning, grid and wire attachment, and FIB milling to create an electron transparent membrane in the active region of the device (Fig. 3).

[1] K.L. Bunker et al., Electronic Device Failure Analysis, 4 (2002), 29. 

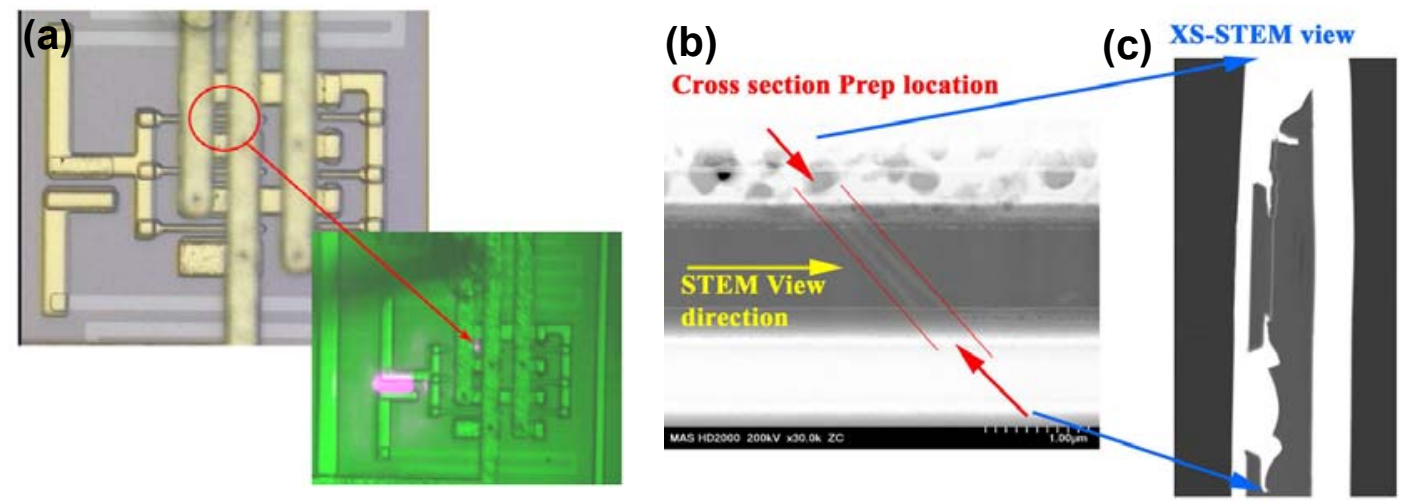

Fig. 1 (a) Optical images of shorted FET indicating the areas of interest. (b) Plan view STEM image of FET cross-sectional preparation location. (c) Cross-sectional sample lifted from plan view sample.
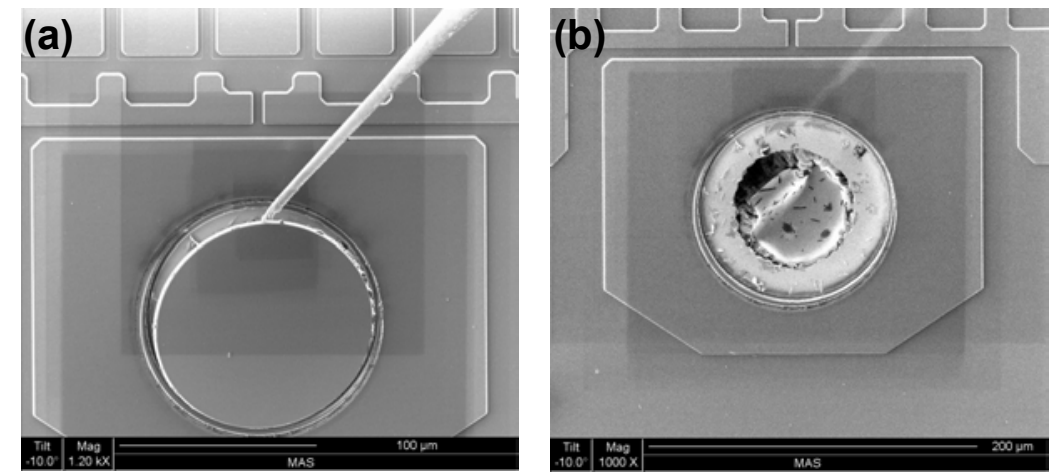

Fig. 2 (a) Optical images of shorted FET indicating the areas of interest. (b) Plan view STEM image of FET cross-sectional preparation location. (b) Cross-sectional sample lifted from plan view sample.
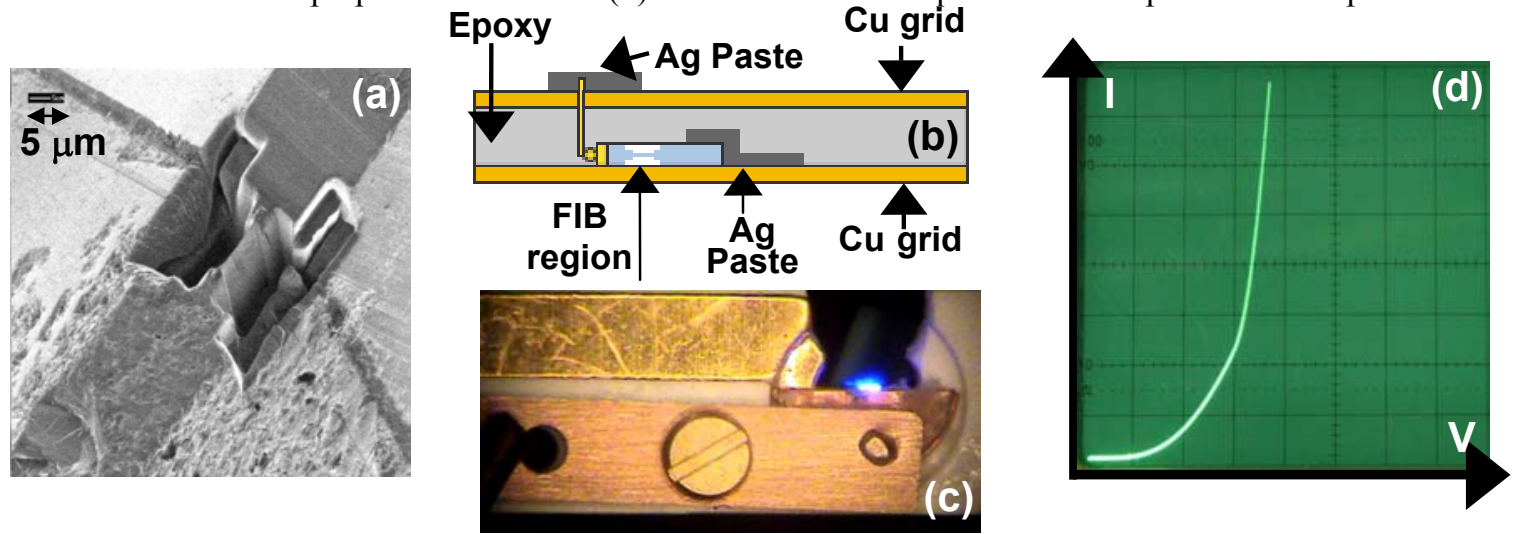

Fig. 3 (a) FIB region of fully prepared sample. (b) Schematic of fully prepared sample for complete STEM analysis. (c) Fully prepared sample mounted in STEM-EBIC holder and emitting under bias. (d) IV curve of a fully prepared InGaN-based LED emitting under bias in the HD-2000 STEM.
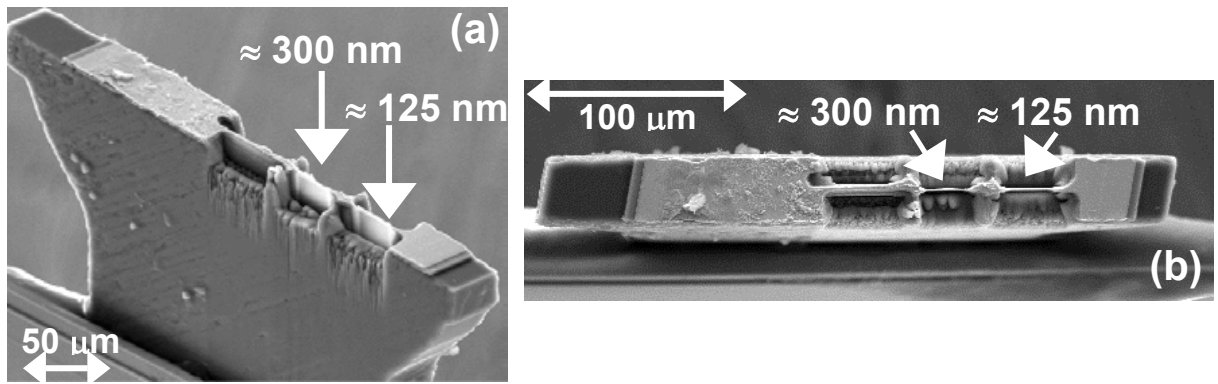

Fig. 4 (a) and (b) Secondary electron images of an InGaN-based multiple quantum well light emitting diode for $\mathrm{CL}$ analysis after FIB thinning. A thin section of $\sim 125 \mathrm{~nm}$ and a thin section of $\sim 300 \mathrm{~nm}$ were created. 